\title{
Quantitative assessment of the retinal microvasculature using optical coherence tomography angiography
}

Zhongdi Chu

Jason Lin

Chen Gao

Chen Xin

Qinqin Zhang

Chieh-Li Chen

Luis Roisman

Giovanni Gregori

Philip J. Rosenfeld

Ruikang K. Wang 


\title{
Quantitative assessment of the retinal microvasculature using optical coherence tomography angiography
}

\author{
Zhongdi Chu, ${ }^{\text {a }}$ Jason Lin, ${ }^{\mathrm{a}}$ Chen Gao, ${ }^{a}$ Chen Xin, ${ }^{\text {a }}$ Qinqin Zhang, ${ }^{\text {a }}$ Chieh-Li Chen, ${ }^{\text {a }}$ Luis Roisman, ${ }^{b}$ \\ Giovanni Gregori, ${ }^{b}$ Philip J. Rosenfeld, ${ }^{b}$ and Ruikang K. Wang ${ }^{a, \star}$ \\ aUniversity of Washington, Department of Bioengineering, 3720 Northeast 15th Avenue, Seattle, Washington 98006, United States \\ bUniversity of Miami Miller School of Medicine, Bascom Palmer Eye Institute, 900 Northeast 17th Street, Miami, Florida 33136, United States
}

\begin{abstract}
Optical coherence tomography angiography (OCTA) is clinically useful for the qualitative assessment of the macular microvasculature. However, there is a need for comprehensive quantitative tools to help objectively analyze the OCT angiograms. Few studies have reported the use of a single quantitative index to describe vessel density in OCT angiograms. In this study, we introduce a five-index quantitative analysis of OCT angiograms in an attempt to detect and assess vascular abnormalities from multiple perspectives. The indices include vessel area density, vessel skeleton density, vessel diameter index, vessel perimeter index, and vessel complexity index. We show the usefulness of the proposed indices with five illustrative cases. Repeatability is tested on both a healthy case and a stable diseased case, giving interclass coefficients smaller than 0.031 . The results demonstrate that our proposed quantitative analysis may be useful as a complement to conventional OCTA for the diagnosis of disease and monitoring of treatment. $\odot$ The Authors. Published by SPIE under a Creative Commons Attribution 3.0 Unported License. Distribution or reproduction of this work in whole or in part requires full attribution of the original publication, including its DOI. [DOI: 10.1117/1.JBO.21.6.066008]
\end{abstract}

Keywords: optical coherence tomography; optical coherence tomography angiography; vascular quantification; vessel area density; vessel skeleton density; vessel diameter index; vessel perimeter index; vessel complexity index.

Paper 160174R received Mar. 19, 2016; accepted for publication May 26, 2016; published online Jun. 10, 2016.

\section{Introduction}

Optical coherence tomography angiography (OCTA) was recently introduced for the imaging of microvascular networks in the human eye. ${ }^{1}$ Recent investigations using OCTA have been mainly focused on showcasing its applications in visualizing the flow characteristics of microvascular diseases within the macula, such as age-related macular degeneration (AMD) ${ }^{2}$ and diabetic retinopathy (DR), ${ }^{3,4}$ the two leading causes of blindness worldwide. ${ }^{5,6}$ In addition, OCTA has documented microvascular abnormalities in other macular diseases such as branch retinal vein occlusion (BRVO), central retinal vein occlusion, ${ }^{7}$ and macular telangiectasia type 2 (MacTel2) ${ }^{8}$ Compared with conventional dye-based angiography, OCTA offers a safer, faster, and more cost effective alternative for ocular imaging of the macular vasculature. Overall, the combination of OCT and OCTA can present integrated structural and flow information of the human eye in vivo, opening new opportunities for both qualitative and quantitative analysis of ocular diseases.

Optical microangiography (OMAG) ${ }^{1}$ is one of the many OCTA approaches that utilizes the intrinsic properties of particles' (e.g., red blood cells) motion, to highlight the contrast between signals due to red blood cells (RBCs) and signals due to static tissues. Unlike other OCTA approaches such as speckle variance, ${ }^{9}$ split-spectrum amplitude decorrelation angiography, ${ }^{10}$ and phase variance, ${ }^{11}$ OMAG is able to harness motion information to the fullest extent by exploiting both amplitude and phase information contained within the OCT signals. Therefore, OMAG is able to

*Address all correspondence to: Ruikang K. Wang, E-mail: wangrk@uw.edu produce OCT angiography with better vascular connectivity, higher signal-to-noise ratio, and higher sensitivity to capillary blood flows. ${ }^{12}$ Consequently, OMAG is being established as a valuable tool for the investigation of ocular diseases both qualitatively ${ }^{13}$ and quantitatively. ${ }^{14}$

To objectively assess the OCT angiograms, a number of studies have introduced vessel area density (VAD), ${ }^{15,16}$ vessel skeleton density (VSD), ${ }^{15,17}$ and nonperfusion area. ${ }^{16}$ These parameters have been reported useful in the analyses of disease progression in DR and AMD. While promising, there are limitations when evaluating indices individually. VAD provides information about the function of vascular network by calculating the percentage of area occupied by vessels on OCTA images. Thus, a change in VAD is coupled with changes in both vessel length and vessel size. Since the decrease of microvascular perfusion is sometimes accompanied with vessel dilation, VAD alone is only capable of providing partial information about vascular abnormalities. Similarly, VSD falls short because it only evaluates the vessel length without taking the vessel size information into account. Moreover, compared with fluorescein angiography (FA), it is difficult for OCTA to convey information about vascular leakage since it detects the existence of moving particles while FA senses the diffusion of injected dyes into the extravascular space. To our best knowledge, there has not been an attempt to extract vascular information other than vessel density from OCT angiograms.

In this study, we introduce a comprehensive quantitative OMAG analysis for OCTA vascular images. We propose to use the following metrics to quantitatively describe the OCTA images: VAD, VSD, vessel diameter index (VDI), vessel 
perimeter index (VPI), and vessel complexity index (VCI). We illustrate the usefulness of these metrics by using five clinical cases. We also assess the repeatability of these metrics by using OCTA scans captured from the same patients at different times. With our comprehensive quantitative OMAG strategy, we aim to provide clinicians and physicians with a rapid strategy for interpreting OCT angiograms from multiple perspectives and to help simplify the process of identifying pathological vascular features so as to enable the objective assessment of disease progression and treatment monitoring.

\section{Method}

An automated tool with a user interactive interface was developed to quantify the abnormalities in the retinal vasculature from multiple perspectives. Included within this tool are the quantitative parameters of VAD, VSD, VPI, VDI, and VCI. To demonstrate the usefulness of these parameters, we used OMAG en face images generated by using both spectral domain OCT (SD-OCT) and swept-source OCT (SS-OCT) angiography prototype instruments.

SD-OCT data was acquired using a Cirrus HD 5000 SDOCT angiography system prototype (Carl Zeiss Meditec Inc., Dublin, California), with an 840-nm central wavelength, $45-\mathrm{nm} \Delta \lambda$ and a $68-\mathrm{KHz}$ A-scan rate. The axial resolution is $\sim 7 \mu \mathrm{m}$ while lateral resolution is $\sim 15 \mu \mathrm{m}$. The scanning protocol in this system provided a field of view (FOV) of $2.4 \mathrm{~mm} \times 2.4 \mathrm{~mm}$, with 245 lines in each B-scan and 245 locations in the slow axis (where four repeated B-scans were acquired at each location). Equipped with FastTrac ${ }^{\mathrm{TM}}{ }^{18}$ technology, a custom sized montage scan could be acquired to give a widefield image with high resolution and high definition. In this study, customized scans consisting of nine motion-tracked smaller cubes, with a total FOV of $6.72 \mathrm{~mm} \times 6.72 \mathrm{~mm}$, were collected from each subject.

SS-OCT data were acquired using a modified CIRRUS prototype containing a swept source laser provided by Carl Zeiss Meditec Inc., which had a 1050-nm central wavelength, $100-\mathrm{nm} \Delta \lambda$ and $100-\mathrm{kHz}$ A-scan rate. The axial resolution is $\sim 5 \mu \mathrm{m}$ while lateral resolution is $\sim 14 \mu \mathrm{m}$. SS-OCT provided a FOV of $3 \mathrm{~mm} \times 3 \mathrm{~mm}$ with 300 A-lines per B-scan and a total of $300 \mathrm{~B}$-scans centered at the fovea. To achieve OMAG imaging of the vascular networks, a previously described repeated B-scan protocol was implemented to acquire the volumetric OCTA data. ${ }^{13,18}$

All participants in this study were consented before OCTA imaging, following the tenets of Declaration of Helsinki, in compliance with the Health Insurance Portability and Accountability Act. The study was approved by the Institutional Review Board of the University of Washington (UW), Seattle, as well as the University of Miami Miller School of Medicine, Miami. All SD-OCT scans were obtained at the University of Washington Eye Institute, while all SS-OCT scans were obtained at the Bascom Palmer Eye Institute, University of Miami Miller School of Medicine. Subjects with the diagnosis of DR and BRVO were prospectively enrolled at the UW Medicine Eye Institute, and subjects with diagnosis of MacTel2 were enrolled in the MacTel project and imaged at the Bascom Palmer Eye Institute.

Figure 1 shows a flowchart providing an overview of the processes involved in our proposed quantitative approach to OCTA metrics. First, a two-dimensional en face OCTA/OMAG image [Fig. 2(a)] is generated from the volumetric data using a semiautomatic segmentation software. ${ }^{19}$ This image is processed into a binary image in MATLAB (R2015b, MathWorks, Inc.) using a global threshold, hessian filter, ${ }^{20}$ and adaptive threshold. ${ }^{15}$ The global threshold is determined by the noise level in the foveal avascular zone (FAZ) that is selected with a user interactive interface by asking the operator to click on the center and edge of FAZ. This global threshold is then applied to the entire image. Then, hessian filter and adaptive threshold are combined to generate a binary vessel map [Fig. 2(b)]. In doing so, the information of vessel area can be obtained. Second, a skeletonized vessel map is created [Fig. 2(c)], where every vessel, regardless of its size or diameter, is represented by a single pixel line. This way, the information of vessel length can be obtained. Last, the vessel perimeter or the edges of vessels are detected and contoured as the vessel perimeter map [Fig. 2(d)] to give the information about vessel perimeter. All five quantitative indices are calculated based on these three binary vessel maps, i.e., vessel area map, skeletonized vessel map, and vessel perimeter map. Note that there are only two values, 1 and 0 , in the binary maps where 1 represents white pixel (i.e., existence of blood vessel) and 0 the black pixel (i.e., absence of blood vessel).

VAD is calculated as a unit less ratio of the total image area occupied by the vasculature to the total image area in the binary vessel maps

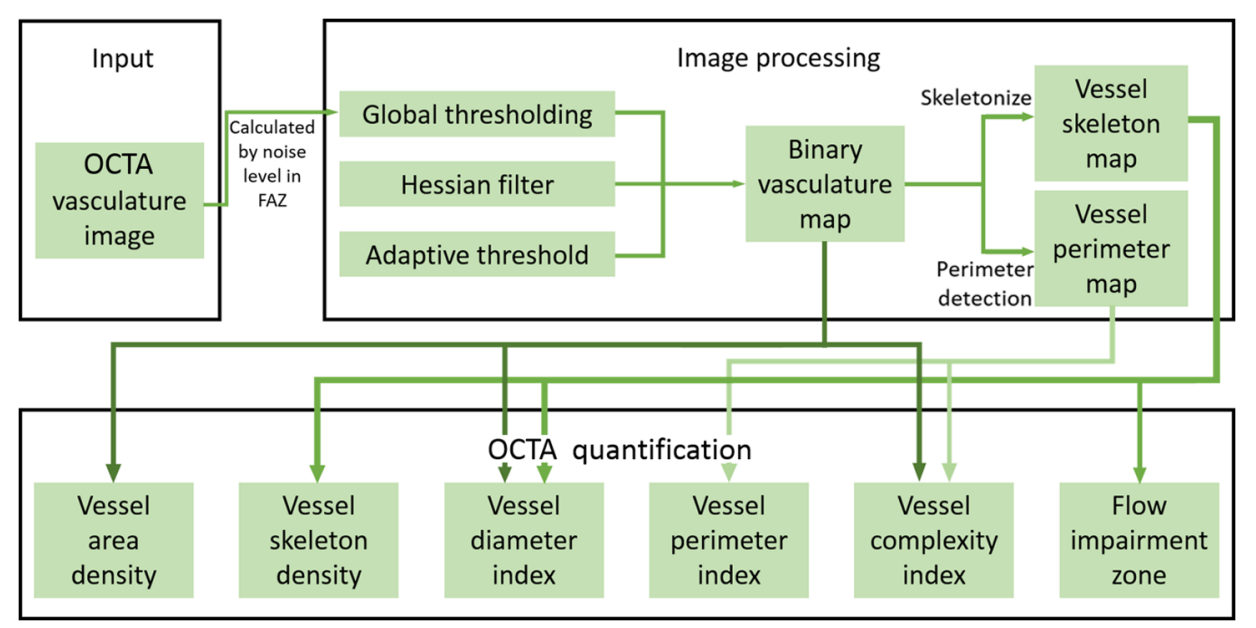

Fig. 1 Overview of the quantitative OMAG algorithm. 

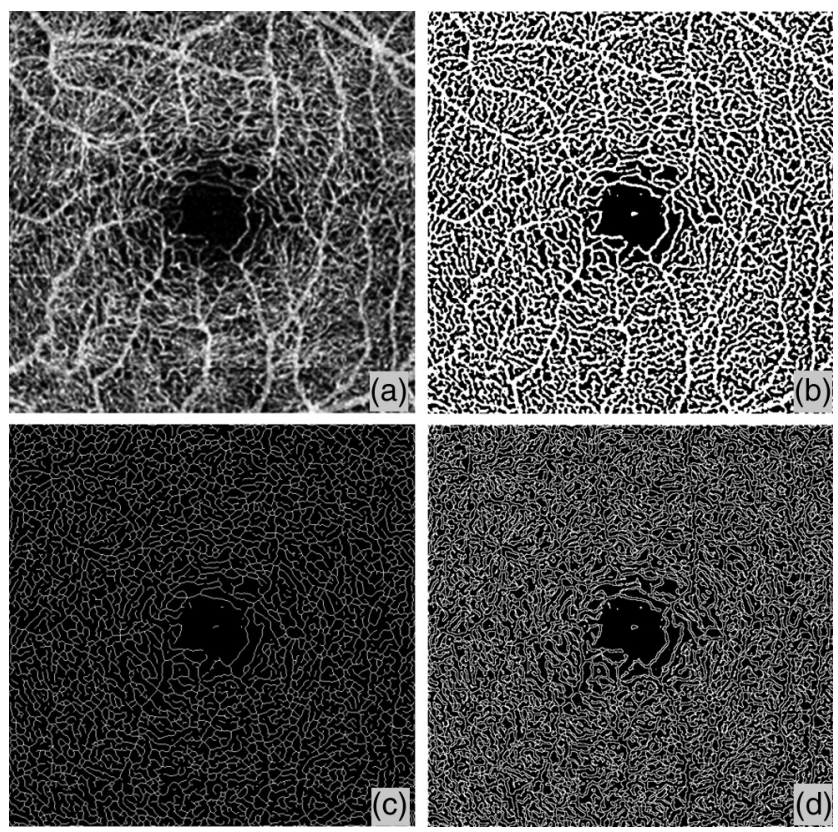

Fig. 2 Representative OMAG images to illustrate the quantitative analysis algorithm. (a) Original en face OMAG image. (b) Vessel area map, a binarized vasculature image using hessian filter and adaptive threshold. This image is used for VAD, VDI, and VCI quantification. (c) Vessel skeleton map, which is obtained by iteratively deleting the pixels in the outer boundary of the vessel area map until one pixel remained along the width direction of the vessels. This image is used for VSD and VDI quantification. (d)Vessel perimeter map, which is obtain by detecting the edge of vessels in the vessel area map and deleting pixels that are not on the edge of vessels. This image is used for VPI and $\mathrm{VCl}$ quantification.

$\mathrm{VAD}=\frac{\sum_{i=1, j=1}^{n} A_{(i, j)}}{\sum_{i=1, j=1}^{n} X_{(i, j)}}$

where $A(i, j)$ represents the pixels occupied by vessel area [white pixels on Fig. 2(b)] and $X(i, j)$ all the pixels in the vessel area map [all pixels on Fig. 2(b)]. $(i, j)$ are the pixel coordinates in the OCTA image (assuming with a size of $n \times n$ pixel array). With this definition, VAD provides the best estimate of real vessel density as it takes both vessel length and vessel diameter into consideration. However, a complication would be that VAD remains unchanged when there are decreased perfusion and vessel dilation happening at the same time, potentially resulting in false negatives on the vascular abnormality. Furthermore, other than computing a single VAD for the whole OCT angiogram, we could also produce a spatially resolved VAD map. A down sampled block map was generated with $25 \times 25$ (pixels) block size, the map was resized to original size and Gaussian filtered with a $3 \times 3$ (pixels) kernel. All maps were generated in the same fashion.

VSD is calculated as the ratio of the length occupied by the blood vessels to the total area in the skeletonized vessel map

$\mathrm{VSD}=\frac{\sum_{i=1, j=1}^{n} S_{(i, j)}}{\sum_{i=1, j=1}^{n} X_{(i, j)}}$,

where $S(i, j)$ represents the pixels registered as vessel length [white pixels on Fig. 2(c)] and $X(i, j)$ represents all the pixels in the skeletonized vessel map [all pixels on Fig. 2(c)]. The unit

for VSD is pixel/pixel. ${ }^{2}$ VSD quantifies the vessel density by only considering whether or not the vessel exists, which is simply an assessment of the vessel length density regardless of the vessel diameters. Since every vessel is presented as a single pixel line, large vessels and small capillaries contribute equally to the VSD quantification. Consequently, compared with VAD, VSD would be more sensitive to the perfusion changes at the capillary levels.

VDI is calculated using both the vessel area map and the skeletonized vessel map to yield the averaged vessel caliber as follows:

$\mathrm{VDI}=\frac{\sum_{i=1, j=1}^{n} A_{(i, j)}}{\sum_{i=1, j=1}^{n} S_{(i, j)}}$,

where $A(i, j)$ represents all the pixels registered as vessel area [white pixels on Fig. 2(b)] and $S(i, j)$ represents the pixels registered as vessel length [white pixels on Fig. 2(c)]. The unit of VDI is pixel and can be converted into micron by multiplying VDI with pixel size, all VDI data in this article has been converted into microns. VDI calculates the averaged vessel caliber within the image; it does not reflect the change in vessel density. VDI presents the vessel size information regardless of the vessel length; therefore, it is sensitive to vascular dilation in the OCTA images. With the VDI map, localized vascular dilation would be easily identified and serve as a marker of vascular abnormalities.

VPI is calculated using the vessel perimeter map as the ratio of the vessel perimeter to the total area of the OCTA image

$\mathrm{VPI}=\frac{\sum_{i=1, j=1}^{n} P_{(i, j)}}{\sum_{i=1, j=1}^{n} X_{(i, j)}}$,

where $P(i, j)$ represents all the pixels within vessel perimeters [white pixels on Fig. 2(d)] and $X(i, j)$ represents all the pixels in the vessel perimeter map [Fig. 2(d)]. The unit for VPI is pixel/ pixel. ${ }^{2}$ Contrary to VSD, VPI uses the perimeter to present every vessel, which includes both the length and the diameter, similar to VAD.

VCI is calculated using both the vessel perimeter map and the vessel area map, defined as

$\mathrm{VCI}=\frac{\left(\sum_{i=1, j=1}^{n} P_{(i, j)}\right)^{2}}{4 \pi \sum_{i=1, j=1}^{n} A_{(i, j)}}$,

where $P(i, j)$ represents all the pixels enclosed by vessel perimeters [white pixels on Fig. 2(d)] and $A(i, j)$ represents all the pixels of the vessel area [white pixels on Fig. 2(b)]. VCI is also a unit less parameter and derived from the digital image processing discipline. ${ }^{21}$ With the vessel complexity map, specific locations where the vascular morphology is less complicated could be identified. In most cases, decreased perfusion would result in less complex vasculature. To the best of our knowledge, this is the first time VCI has been introduced to quantify morphological characteristics of the retinal vasculature, though fractal dimension has been previously used to describe vascular tortuosity. ${ }^{15}$ To better illustrate the concept of VCI, an example study was conducted to test the robustness of this quantitative parameter. As shown in Fig. 3, five images were selected as examples for the calculation of VCI. Both binary area images and perimeter images are shown in Figs. 3(a)-3(j), and the VCI results for each image are plotted in Fig. 3(k), with the exact 

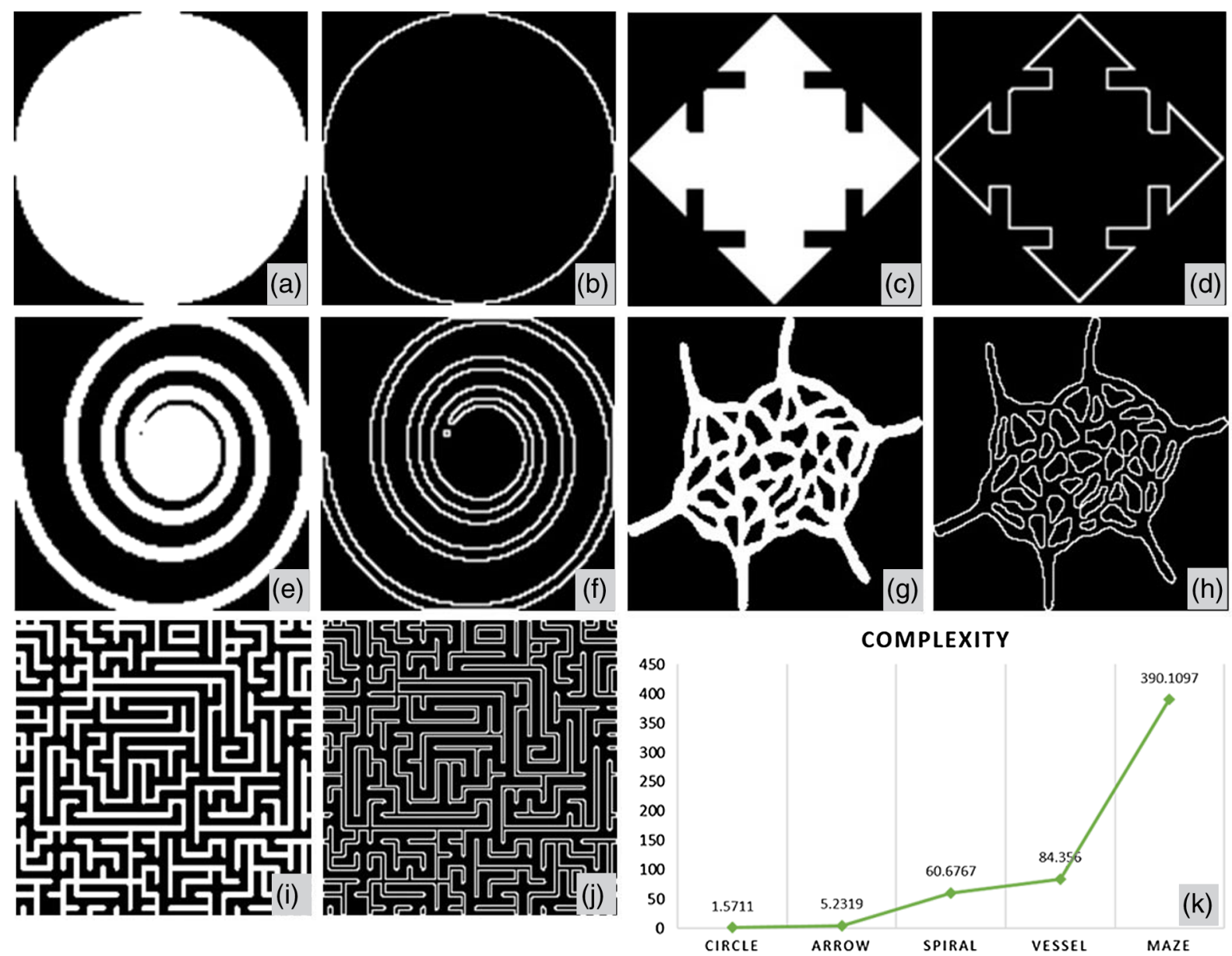

Fig. 3 Demonstration of $\mathrm{VCl}$ quantification. (a)-(j) are area images (a), (c), (e), (g), and (i) and perimeter images (b), (d), (f), (h), and (j), with increasing visual complexity. Plot (k) shows their corresponding quantification of $\mathrm{VCl}$.

values denoted above each data point. We find a positive relationship between VCI and its visual complexity; as VCI increases, the visual complexity of the image also increases. Theoretically the VCI value should be 1 for a circle; however, due to the discrete nature of computing, computed VCI value for a circle would always be larger than 1 , depends on sampling rate. All computed VCIs have been normalized according to this 1.5711 to 1 ratio.

\section{Results}

Five illustrative cases are shown to demonstrate the usefulness of all the quantitative metrics that we propose.

\subsection{Retinal Vasculature and Quantitative Maps in Normal Case}

The three-dimensional (3-D) nature of OCT allows for segmentation of vascular plexi located at different physiological depths within the retina. Using a validated semiautomatic segmentation software ${ }^{19}$ we separated the retinal microvasculature into three distinct physiological layers: the superficial vascular network encompassing from ganglion cell layer to inner plexiform layer, the deep vascular network including the outer plexiform layer, and the avascular outer retinal layer extending from outer nuclear layer to retinal pigment epithelium as shown in Fig. 4(b). We excluded the retinal nerve fiber layer in the following examples since the vasculature in this layer is morphologically different from the vasculature in the deeper retinal layers, especially for large FOV OCTA scans. ${ }^{22}$ However, this layer can be included in the analysis if needed. Figure 4(a) shows the en face OCTA image with maximum projection, with the color scheme for segmented vascular plexus shown in Fig. 4(b).The vessel density map, vessel diameter map, and vessel complexity map are shown in Figs. 4(c)-4(e). Figures 4(f)-4(h) were obtained by combining Figs. 4(c)-4(e) with the vessel map [Fig. 2(b)], which would make it easier to localize the particular vessels of interests. Good correlations can be observed in these three maps with the anatomic features of the retinal vasculature. For both vessel density map and vessel diameter map, large vessels in the superficial layer appear red, which represents higher density/diameter; in the vessel complexity map, large vessels are blue since they are less complex in morphology compared to capillaries. Figure 4(h) shows the overlay of vessel complexity map and deep retinal vascular layer en face maximum projection image, where we have observed that the red regions in the vessel complexity map correspond to the radial fan-like capillaries that originate from vertical anastomosis connecting superficial and deep network.

With the algorithm, VAD, VSD, VPI, VDI, and VCI can all be quantified from the OMAG images. For illustrative purposes, an example of five quantitative indices has been given in Table 1 based on the entire retinal image [Fig. 4(a)]. 

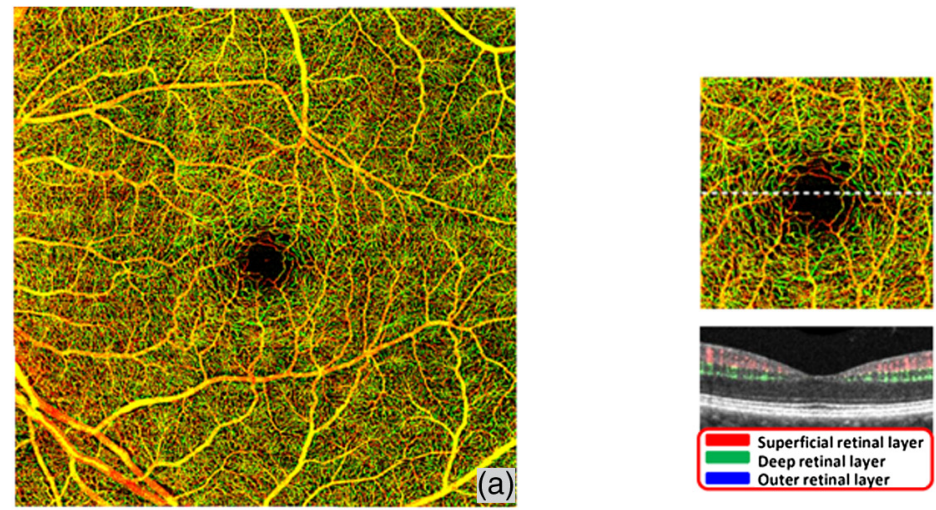

(b)


Vessel Density map with Binary Vessels

Vessel Diameter map with Binary Vessels
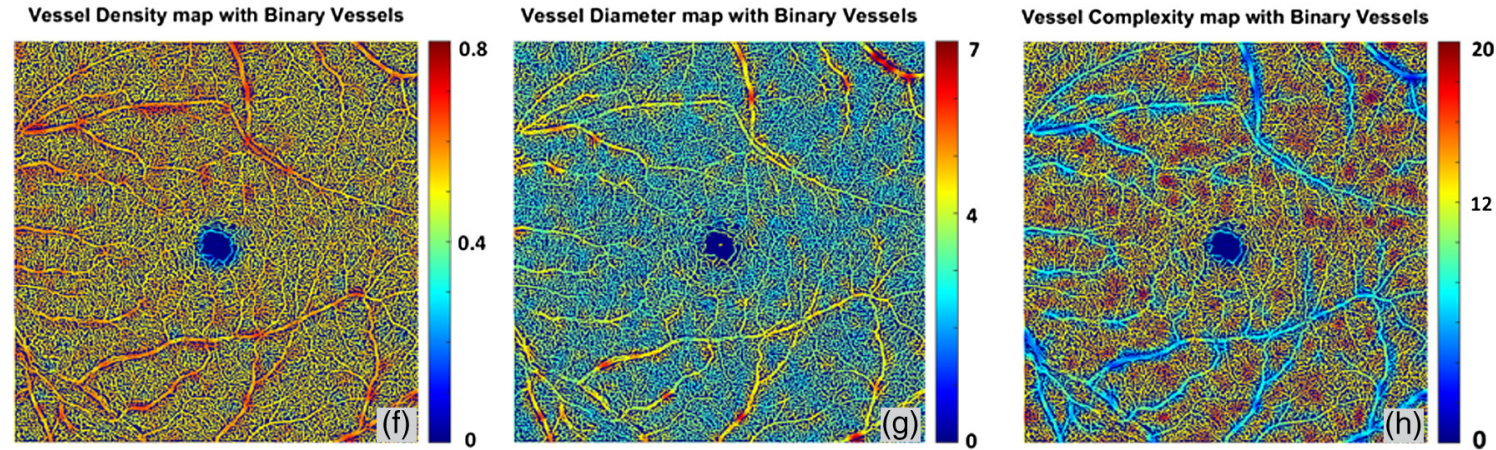

Fig. 4 Quantitative OMAG on a normal case. (a) OMAG en face image with montage scanning protocol, nine smaller cubes are stitched together to achieve a $6.72 \mathrm{~mm} \times 6.72 \mathrm{~mm}$ FOV. (b) Illustration of segmentation and color code strategy. Retinal vasculature was segmented into superficial slab, deep slab, and the avascular outer retina slab. Red represents superficial plexus, green represents deep plexus, and blue for outer retinal slab. All three slabs were integrated together and represented in a color image in (a), excluding retinal nerve fiber layer. (c) Vessel density map. This map shows the VAD of image (a), calculated using a kernel moving across the entire OMAG image. (d) Vessel diameter map. This map shows the local averaged VDI using the same calculation method as (c). (e) Vessel complexity map. This map shows the local averaged $\mathrm{VCl}$ using the same calculation method as (c). (f)-(h) Integration of quantitative maps (c)-(e) with binary vessel area map [Fig. 2(b)].

Table 1 Quantitative analysis of a normal case and a NPDR case.

\begin{tabular}{|c|c|c|c|c|c|c|}
\hline Indices & VDI & VAD & VSD & VPI & $\mathrm{VCl}$ & $\begin{array}{c}\text { Flow } \\
\text { impairment } \\
\text { zone }\left(\mathrm{mm}^{2}\right.\end{array}$ \\
\hline Normal & 24.421 & 0.518 & 0.146 & 0.349 & 17838.540 & 0.264 \\
\hline DR & 24.665 & 0.370 & 0.101 & 0.245 & 12954.770 & 3.450 \\
\hline
\end{tabular}

\subsection{Microvascular Abnormality and Flow Impairment in Diabetic Retinopathy}

Quantitative OMAG analysis of an eye with nonproliferative diabetic retinopathy (NPDR) is shown in Fig. 5. A late phase fluorescein angiographic image shown in Fig. 5(a) corresponds well to the OMAG image shown in Fig. 5(b). The areas of flow impairment are automatically detected according to our vessel skeleton map and superimposed onto OMAG en face image in green [Fig. 5(c)]. For this example, the negation of the vessel skeleton map was first obtained, and then the morphological operations were conducted to close smaller unconnected regions (600 pixels) that come naturally between vessels. The size of this 

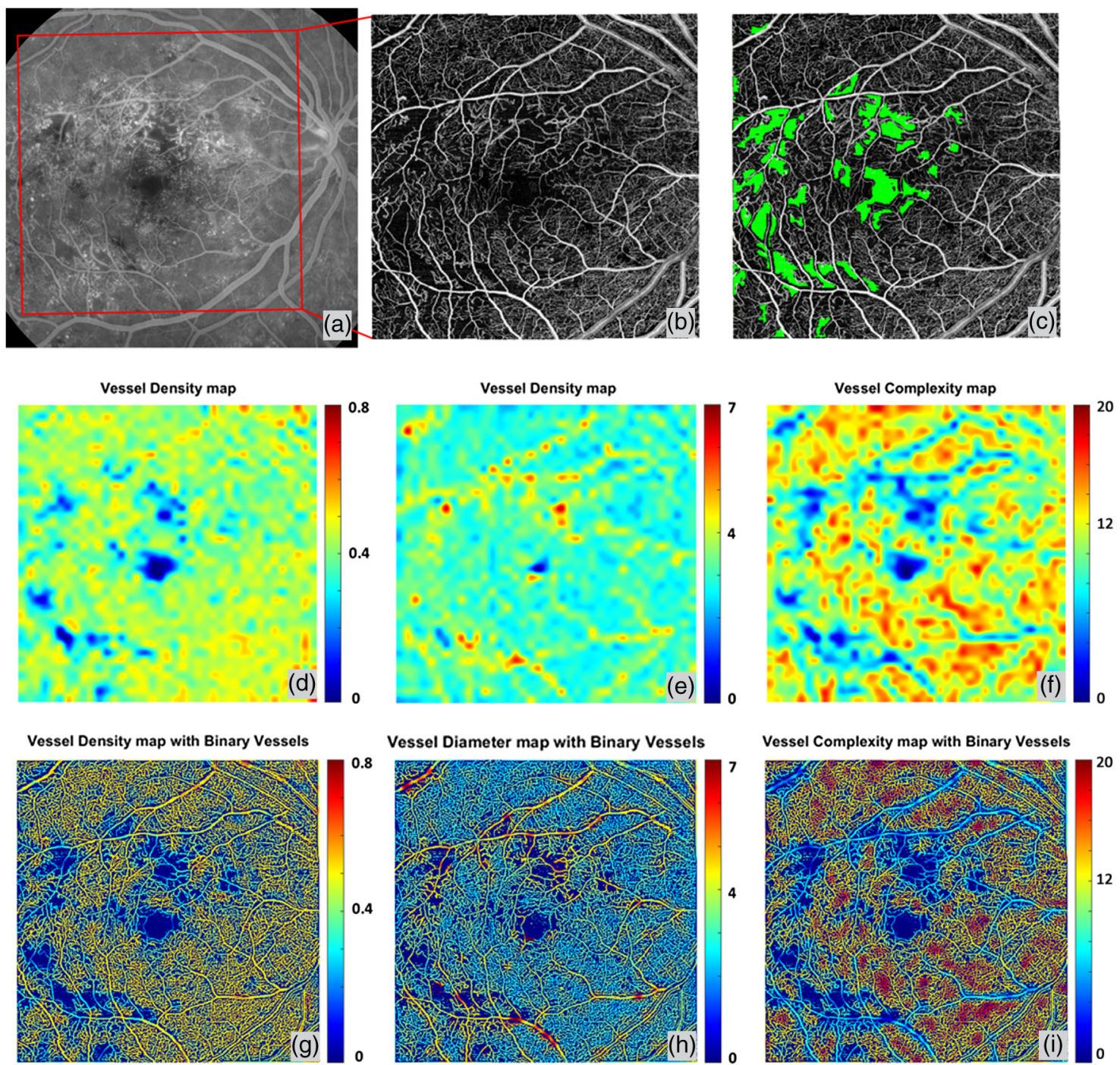

Vessel Diameter map with Binary Vessels

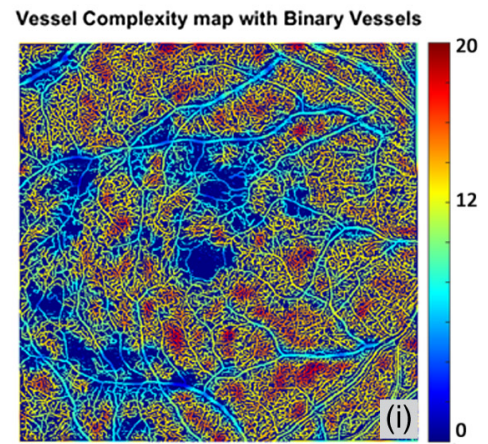

Fig. 5 Quantitative OMAG on a NPDR case. (a) FA image. (b) Whole retina OMAG image, correspond to the red rectangular region in the FA image. Scanning area is $6.72 \mathrm{~mm} \times 6.72 \mathrm{~mm}$. (c) Flow impairment zone quantification. Capillary drop out region was detected from vessel skeleton image and presented in green color, superimposed on (b). (d) Vessel density map. (e) Vessel diameter map. (f) Vessel complexity map. (g)-(i) Integration of quantitative maps (d)-(f) with binary vessel area map.

region was determined according to normal OCTA scans and might differ among different OCT systems. Last, a Gaussian filter (with a kernel size of 3 pixel $\times 3$ pixel) was used to smooth the edges of the detected regions of flow impairment. Vessel density, diameter, and complexity maps have been quantified as shown in Figs. 5(d)-5(f). Figures 5(g)-5(i) show the results by overlying the quantitative maps onto binary vessel area map. It can be observed from the quantitative maps that the regions of capillary flow impairment are often associated with an increase of vessel diameter and a decrease in VAD and vessel complexity, which agrees well with clinical features observed from DR patients. Specific quantitative results are shown in Table 1.

\subsection{Repeatability of Quantitative Optical Microangiography in Branch Retinal Vein Occlusion and Normal Cases}

Figure 6 shows a side-by-side comparison of two scans from the same subject diagnosed with BRVO, but scanned at time points one month apart. This case was clinically diagnosed as stable over the time period as judged by fundus examination, FA and visual acuity. OMAG results demonstrated that the detailed characteristics of BRVO such as vascular dilation and capillary nonperfusion are repeatable between these two scans. Quantitative maps [Figs 6(g)-6(1)] also show great repeatability, where the increase in vessel diameter and decrease in vessel density and vessel complexity are present in regions of the vein occlusion in both scans. VAD, VSD, VDI, VPI, and VCI have also been quantified on two scans. Results show high repeatability as the coefficients of variance are all smaller than 0.031 (Table 2). In doing so, each map was resized to 100 superpixels and repeatability between different scans was quantified based on these 100 samples.

Similarly, two repeated scans of a normal subject (Fig. 7) show great repeatability of not only the OMAG en face images, but also three quantitative maps. Coefficients of variance have also been quantified for five quantitative indices and all are no greater than 0.021 (Table 3). Therefore, our OCTA quantification 

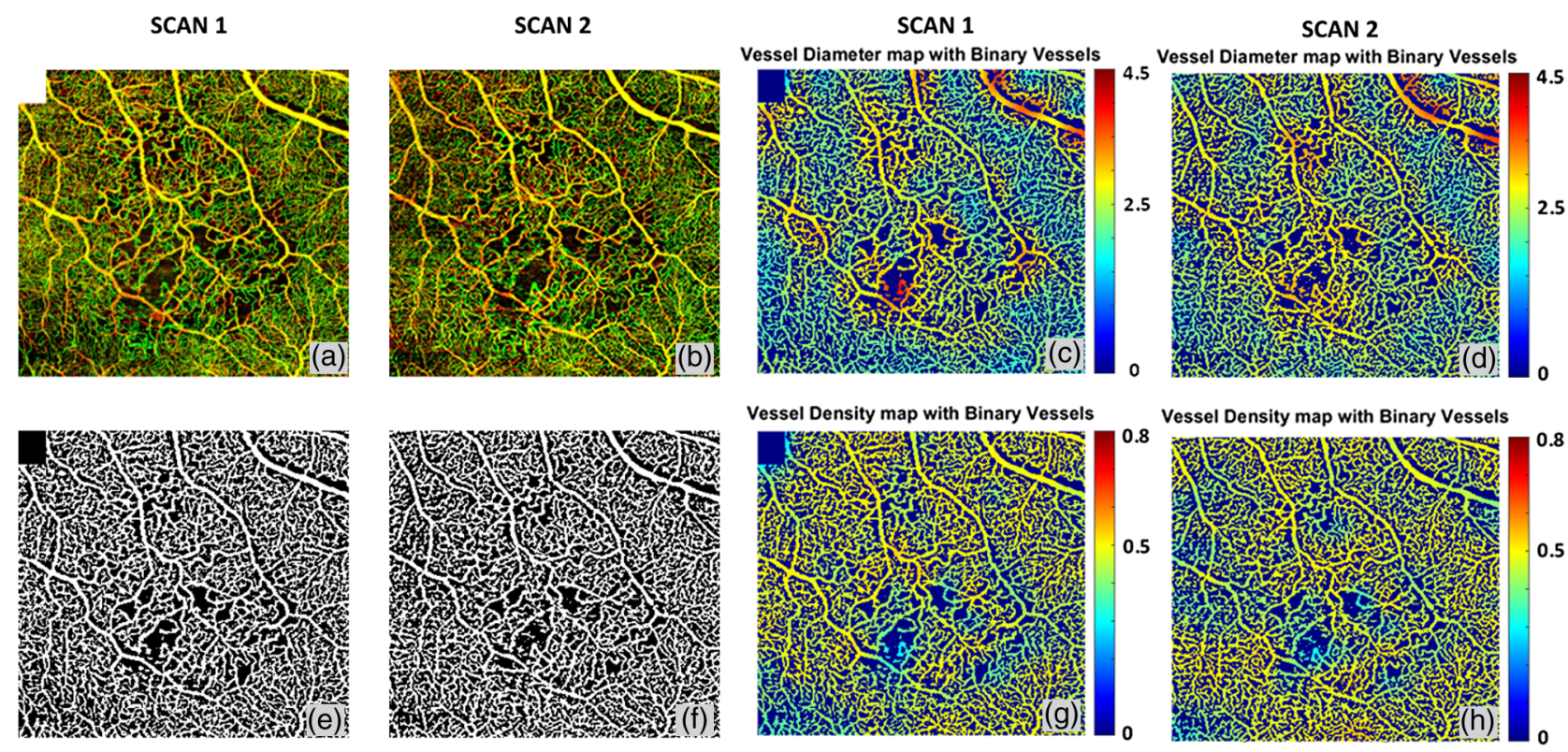

Vessel Density map with Binary Vessels
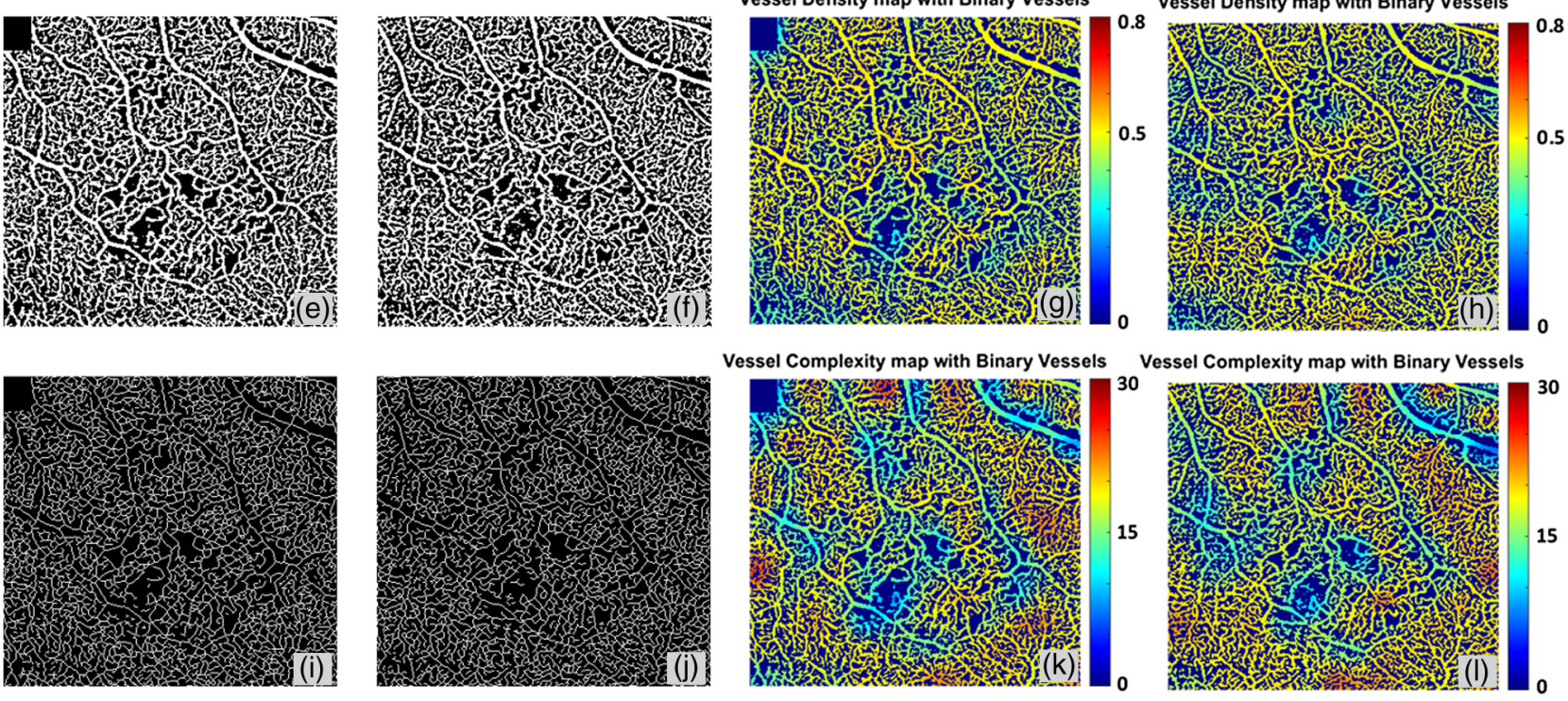

Fig. 6 Quantitative OMAG analysis of a BRVO case with repeated scans. (a) and (d) OMAG image of occluded region, covering a FOV of $4.3 \mathrm{~mm} \times 4.6 \mathrm{~mm}$. (b) and (e) Vessel area map. (c) and (f) Vessel skeleton map. (g) and (j) Vessel density map integrated with vessel area map. (h) and (k) Vessel diameter map integrated with vessel area map. (i) and (I) Vessel complexity map integrated with vessel area map.

Table 2 Quantitative analysis and repeatability test of a BRVO case for two repeated scans.

\begin{tabular}{lccccc} 
& VDI & VAD & VSD & VPI & VCI \\
\hline Scan 1 & 35.300 & 0.449 & 0.175 & 0.395 & 2862.719 \\
Scan 2 & 34.196 & 0.451 & 0.182 & 0.393 & 2827.102 \\
Coefficient of variance & 0.017 & 0.014 & 0.013 & 0.031 & 0.014 \\
\hline
\end{tabular}

algorithm should be useful for monitoring disease progression and treatment response.

Moreover, we have implemented this algorithm in a software package where the operator can flexibly select their regions of interest on the en face image with the following options of e.g., a rectangle, circle, ring, or ellipse. Figure 8 shows an example of our region of interest (ROI) selection, Figure $8(\mathrm{a})$ is the original OMAG en face image, Figure 8(b) shows the quadratic analysis with a ring ROI, and Figs. 8(c)-8(f) shows the circle, rectangle, ellipse, and circle ROI selection, respectively. Table 4 shows the specific quantitative values when different ROIs are selected. If the selection is a ring, it can be divided into four quadrants to enable quadrant specific quantification. Table 5 shows results of quadratic analysis of this BRVO case. Note that in the scans centered at fovea, the operator can choose to center their ring ROI at the fovea centralis.

\subsection{Vascular Dilation in Macular Telangiectasia Type 2}

Figure 9 shows an example of an early stage MacTel 2 case, where the FA image [Fig. 9(a)] shows the signature juxtafoveal leakage that is more prominent temporally than nasally. The en face OMAG image and quantitative maps are given for the entire retina and deep capillary plexus in Figs. 9(b)-9(e) and 9(f)-9(i), respectively. The vessel diameter map and vessel complexity map demonstrate good accordance with the dye leakage on FA, and all demonstrate the telangiectatic changes in the microvasculature. With the dilation of the juxtafoveal microvasculature, local vessel diameter increases and the local vessel complexity decreases significantly, when compared with the relatively normal regions. The comparison between the entire retinal vasculature and deep capillary plexus also validates that the early telangiectatic changes mainly happen in deep capillary plexus $^{23}$ as the quantitative maps manifest more obvious changes. With the segmentation strategy and high resolution OMAG images, a detailed depiction of the deep retinal microvasculature can be produced, as shown in Figs. 9(g)-9(i), which cannot be achieved using traditional FA. Good correlations can be observed between the leaking regions on FA and the 

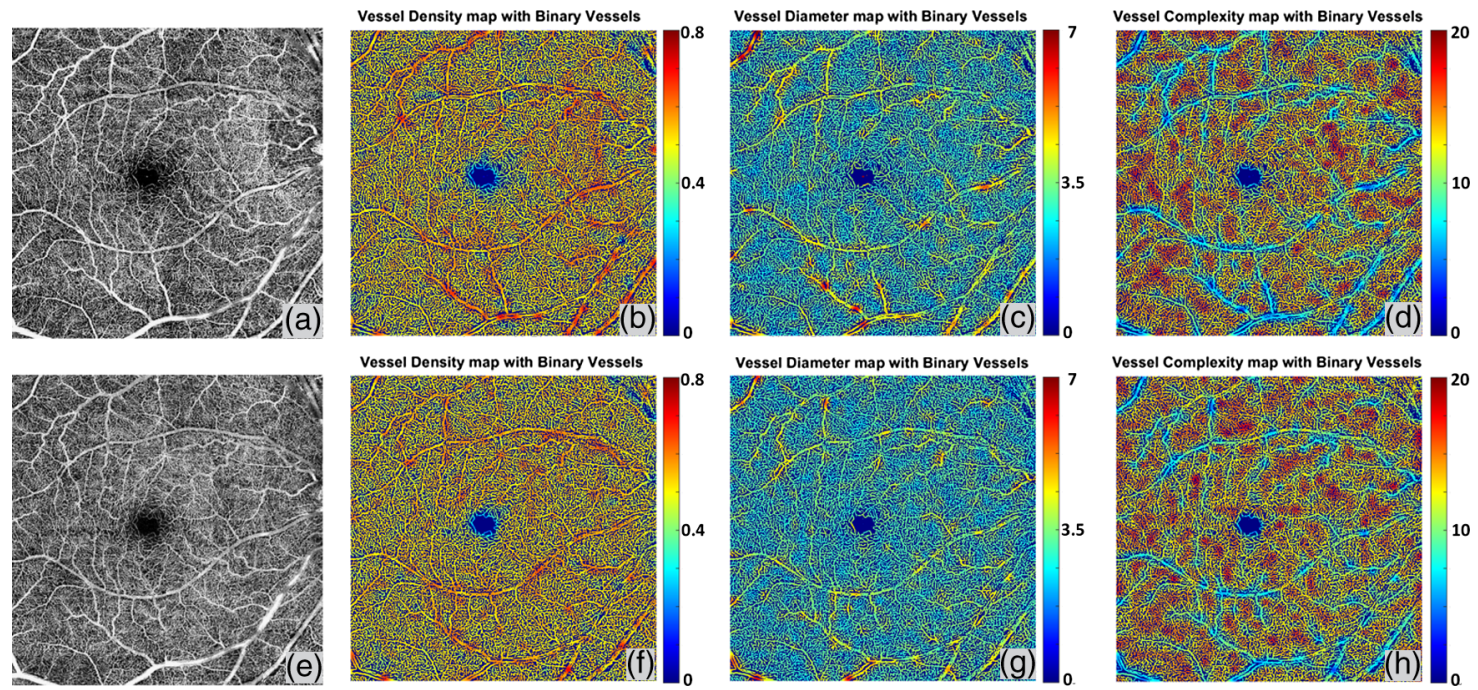

Fig. 7 Repeatability of Quantitative OMAG on a normal case. (a) Whole retina OMAG image of a normal case, covering an area of $6.72 \mathrm{~mm} \times 6.72 \mathrm{~mm}$. (e) Repeated scan of the same subject at same location, whole retina OMAG image. (b) and (f) Vessel density map integrated with the binary vessel area map. (c) and (g) Vessel diameter map integrated with the binary vessel area map. (d) and (h) Vessel complexity map integrated with the binary vessel area map.

Table 3 Repeatability test of a normal case.

\begin{tabular}{lrrrrr}
\hline & & & & & \\
\hline Scan 1 & 23.206 & 0.511 & 0.151 & 0.353 & 19282.771 \\
Scan 2 & 23.619 & 0.512 & 0.149 & 0.351 & 18979.230 \\
Coefficient of variance & 0.018 & 0.009 & 0.021 & 0.011 & 0.017 \\
\hline
\end{tabular}

abnormal regions on the vessel diameter map and the vessel complexity map on the OMAG images. This observation may be clinically important because if true, then quantitative OMAG may present the ability not only to detect the same vascular abnormalities as FA, but also to provide information about the areas of potential leakage without the need for FA. Additional patients are needed to validate this observation.

Figure 10 shows the side-by-side comparison of a normal subject and the MacTel2 subject. Subtle differences can be noticed in the vessel density maps as the MacTel 2 case shows a lower global VAD. Distinct differences can be found on both
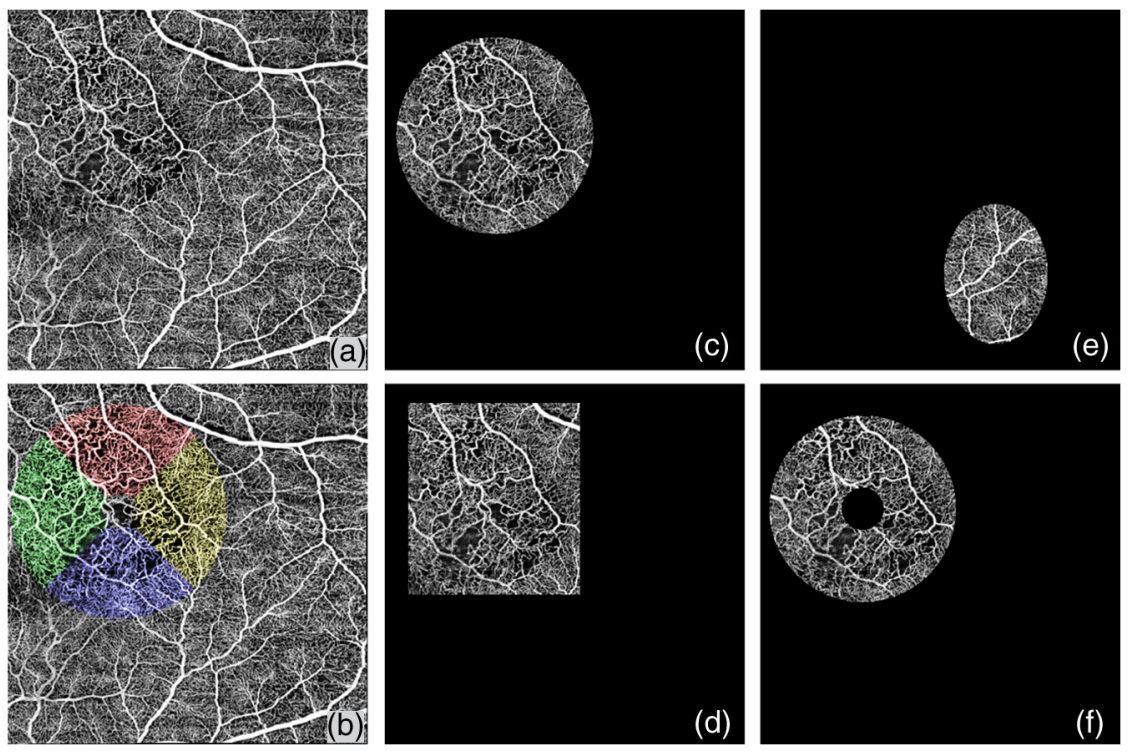

Fig. 8 (a) Illustration of region of interest selection. An original OCTA image with a FOV of $6.72 \mathrm{~mm} \times 6.72 \mathrm{~mm}$. (b) Illustration of quadratic analysis. Red represents superior quadrant, green represents nasal quadrant, blue represents in inferior quadrant, and yellow represents temporal quadrant. (c) ROI selection example of a circle. (d) ROI selection example of a rectangle. (e) ROI selection example of an ellipse. (f) ROI selection example of a circle. 
Table 4 Quantitative analysis of a BRVO case with different choice of region of interest.

\begin{tabular}{lcccccc} 
& VAD & VSD & VPI & VDI & VCl & $\begin{array}{c}\text { ROI size } \\
(\mathrm{mm} \times \mathrm{mm})\end{array}$ \\
\hline Whole image & 0.493 & 0.200 & 0.419 & 32.554 & 7234.772 & 44.890 \\
Circle & 0.491 & 0.195 & 0.418 & 33.272 & 396.946 & 10.521 \\
Rectangle & 0.491 & 0.193 & 0.415 & 33.633 & 457.660 & 11.389 \\
Ellipse & 0.519 & 0.220 & 0.454 & 31.28575 & 62.690 & 3.964 \\
Ring & 0.494 & 0.193 & 0.416 & 33.812 & 285.845 & 8.987 \\
\hline
\end{tabular}

Table 5 Quadratic quantitative analysis of a BRVO case.

\begin{tabular}{lcccccc}
\hline & & & & & & \\
& VAD & VSD & VPI & VDI & VCI & $\begin{array}{c}\text { ROI size } \\
(\mathrm{mm} \times \mathrm{mm})\end{array}$ \\
\hline Superior & 0.493 & 0.197 & 0.411 & 33.942 & 239.2756 & 6.0934 \\
Inferior & 0.473 & 0.202 & 0.424 & 32.034 & 257.6289 & 6.0934 \\
Nasal & 0.478 & 0.200 & 0.424 & 32.838 & 251.9077 & 6.0934 \\
Temporal & 0.489 & 0.194 & 0.413 & 34.292 & 237.8684 & 6.0934 \\
Whole image & 0.493 & 0.200 & 0.419 & 32.554 & 7234.772 & 44.890 \\
\hline
\end{tabular}

vessel diameter maps and vessel complexity maps. The MacTel2 case also shows a significantly increased value in the juxtafoveal temporal and nasal regions on the vessel diameter map when compared with the normal case and an extensively decreased vessel complexity value when compared with the normal eye in the same regions of interest.
Moreover, our five-index quantitative analysis may be useful in the treatment monitoring. Figure 11 shows results from a patient who received monthly injections of a vascular endothelial growth factor (VEGF) inhibitor from January 2015 to March 2015. OCTA scans were performed at each visit with the first scan before treatment and the subsequent two scans performed one month after each treatment. OCTA volume data were segmented into superficial retinal layer, deep retinal layer, and outer retinal layer. En face images were generated for each layer using maximum projection for all three scans. A1-C1 are the superficial retinal layer, deep retinal layer, and outer retinal layer OCT angiograms, respectively, for the first visit before treatment; $\mathrm{A} 2-\mathrm{C} 2$ are for the second visit one month after the first treatment; and A3-C3 are for the third visit one month after the second treatment. For each visit, the deep retinal layer angiograms were chosen for the quantification of vessel density maps, vessel diameter maps, and vessel complexity maps, which are shown in D1-D3, E1-E3, and F1-F3, respectively. By observing these quantitative maps after the first injection, we found that the VDI decreased between the first and second visits, and the VCI has increased. In order to test whether our quantitative analysis has the ability to monitor the changes in vascular abnormalities after treatment, we performed quadrant-specific statistical analysis on this case for all three visits. A 2-mm circle was selected for statistical analysis as showed in Figure 12, and superior, inferior, temporal, and nasal quadrants are labeled in different colors. In each quadrant, all data points were equally divided into ten subsets and the averaged values were used to represent each subset. Therefore, we have ten samples of each quantitative index for each quadrant. The averaged value of each quadrant for each index are shown in Table 6. ANOVA was performed and the VDI showed significant decrease in superior, temporal, and inferior quadrants after treatment while VCI were found significantly increased in temporal and inferior quadrants after treatment. No significant differences were found in any quadrants for VAD, VSD, or VPI. FA was only performed at the first visit therefore we do not have all three corresponding FA images to compare with OCTA

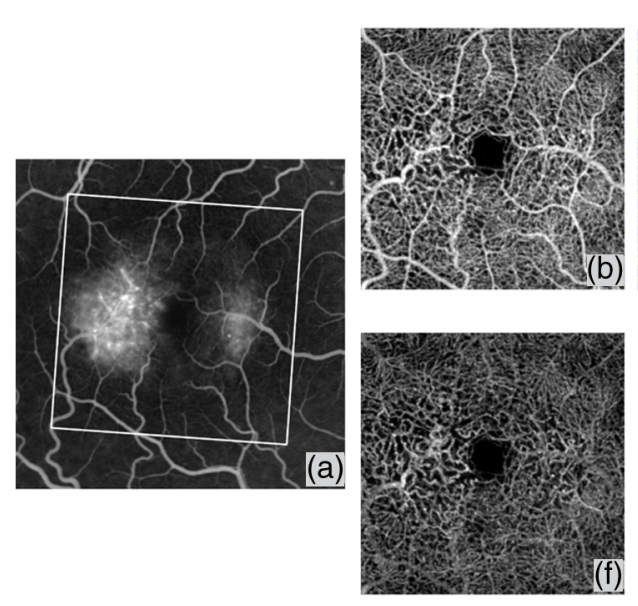

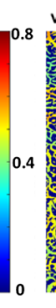
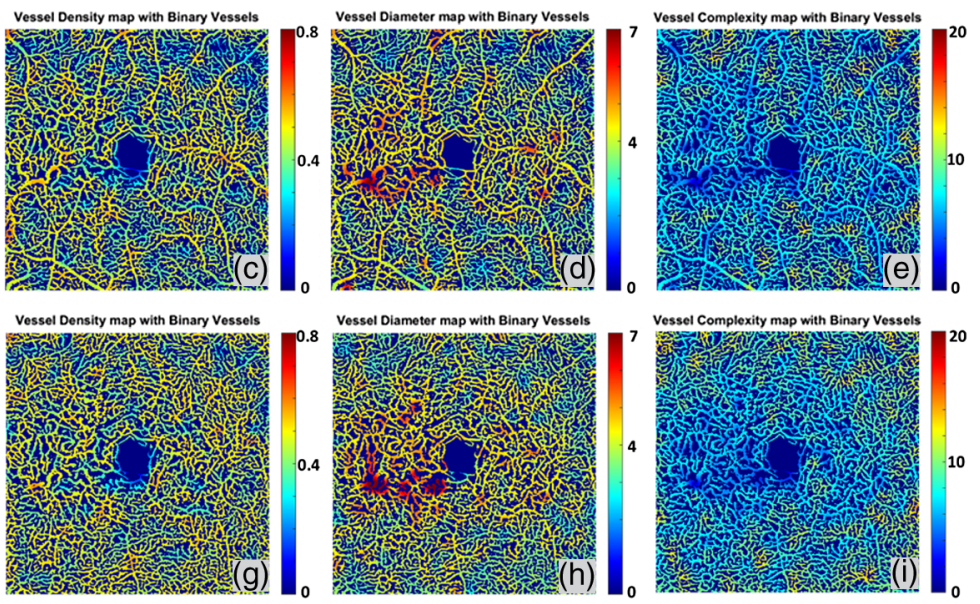

Fig. 9 Quantitative OMAG analysis of a MacTel2 case. (a) FA image of the MacTel2 subject. (b) SS-OCT OMAG image of the whole retina, covering $3 \mathrm{~mm} \times 3 \mathrm{~mm}$ area, corresponding to the white rectangular region in FA image. (c) Vessel density map integrated with vessel area map on the whole retina. (d) Vessel diameter map integrated with vessel area map on the whole retina. (e) Vessel complexity map integrated with vessel area map on the whole retina. (f) OMAG image of the deep capillary plexus. (g) Vessel density map integrated with vessel area map of the deep capillary plexus. (d) Vessel diameter map integrated with vessel area map of the deep capillary plexus. (e) Vessel complexity map integrated with vessel area map of the deep capillary plexus. 

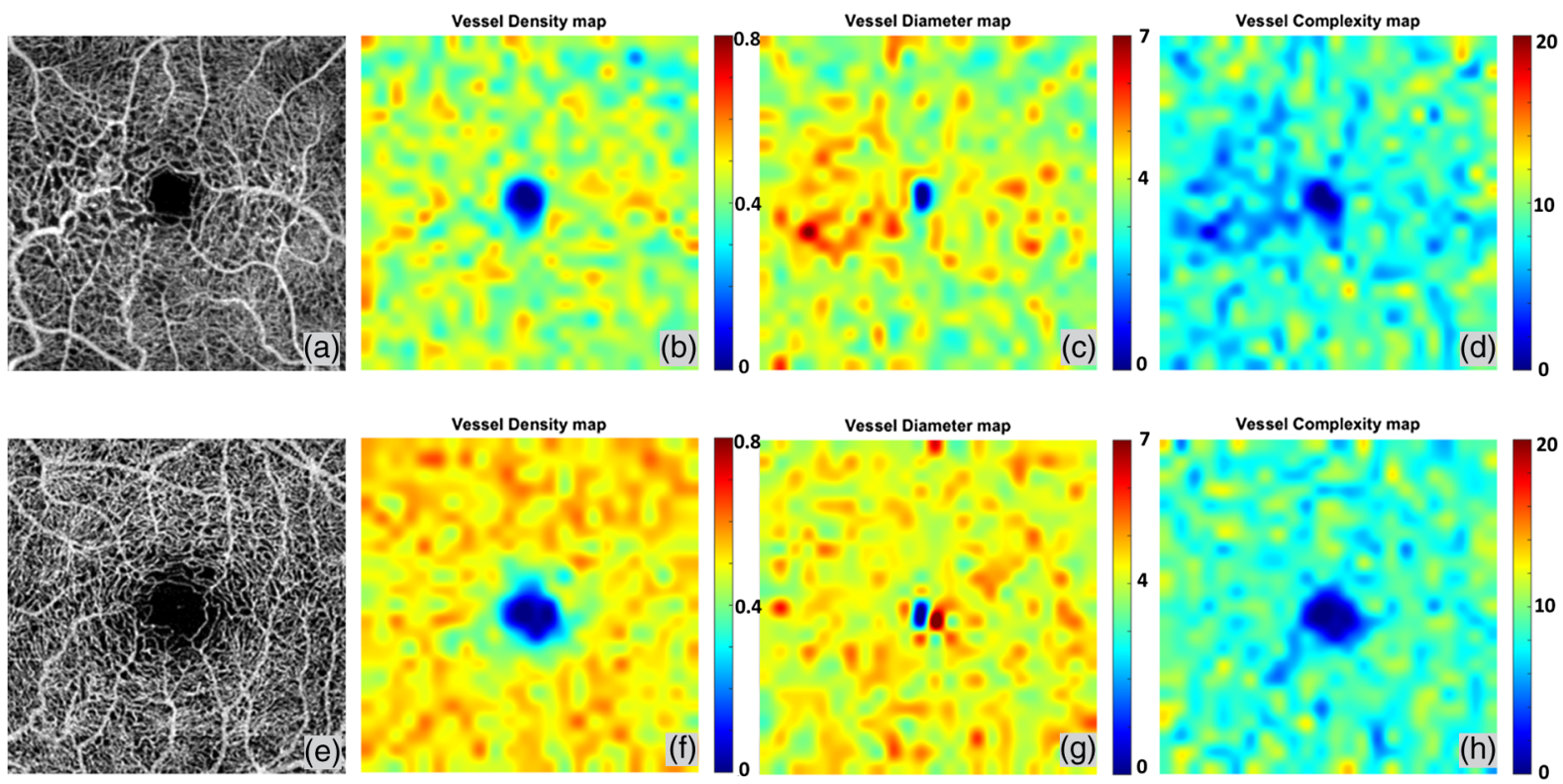

Fig. 10 Quantitative OMAG comparison between the MacTel2 and normal cases. (a) $3 \mathrm{~mm} \times 3 \mathrm{~mm}$ OMAG image of the whole retina of a MacTel2 subject. (b) Vessel density map. (c) Vessel diameter map. (d) Vessel complexity map. (e) $3 \mathrm{~mm} \times 3 \mathrm{~mm}$ OMAG image of the whole retina of a normal subject. (f) Vessel density map. (g) Vessel diameter map. (h) Vessel complexity map.
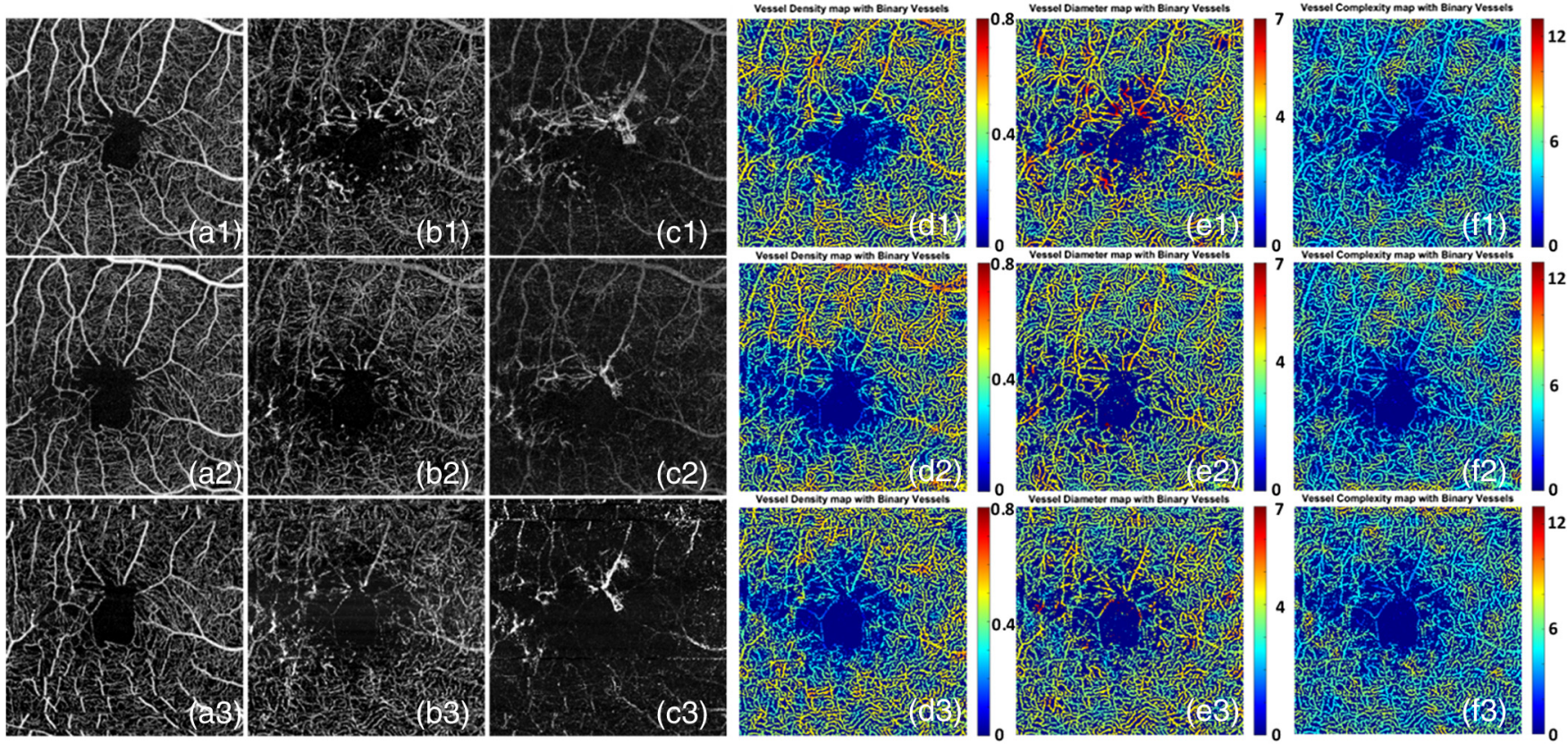

Fig. 11 Three OCTA scans of a patient with MacTel2 monthly apart. a1-a3 shows the superficial retina for the first, second, and third scans, respectively. b1-b3 shows the deep retina for three scans, respectively. c1-c3 shows the outer retina for three scans, respectively. $d 1-d 3$ shows the vessel density map for three scans, respectively and e1-e3 shows the vessel diameter maps for three visits while f1-f3 shows the vessel complexity maps for all three visits.

images. While these results are intriguing, a larger study is clearly needed to fully validate the usefulness of our quantitative analysis for monitoring treatment response after antiVEGF therapy.

\subsection{Building Database for Normal Subjects}

Looking forward, we are in the process of building a normal OCTA database for quantification. We have currently enrolled five normal subjects (age matched) and obtained $6.72 \mathrm{~mm} \times$ $6.72 \mathrm{~mm}$ montage OCTA scans from each of them. OMAG en face images have been produced and used for quantification. Descriptive statistics of normal subjects are shown in Table 7. With the establishment of a normal database, future studies that compare diseased cases with normal cases or that determine disease progression or classification would be feasible. 


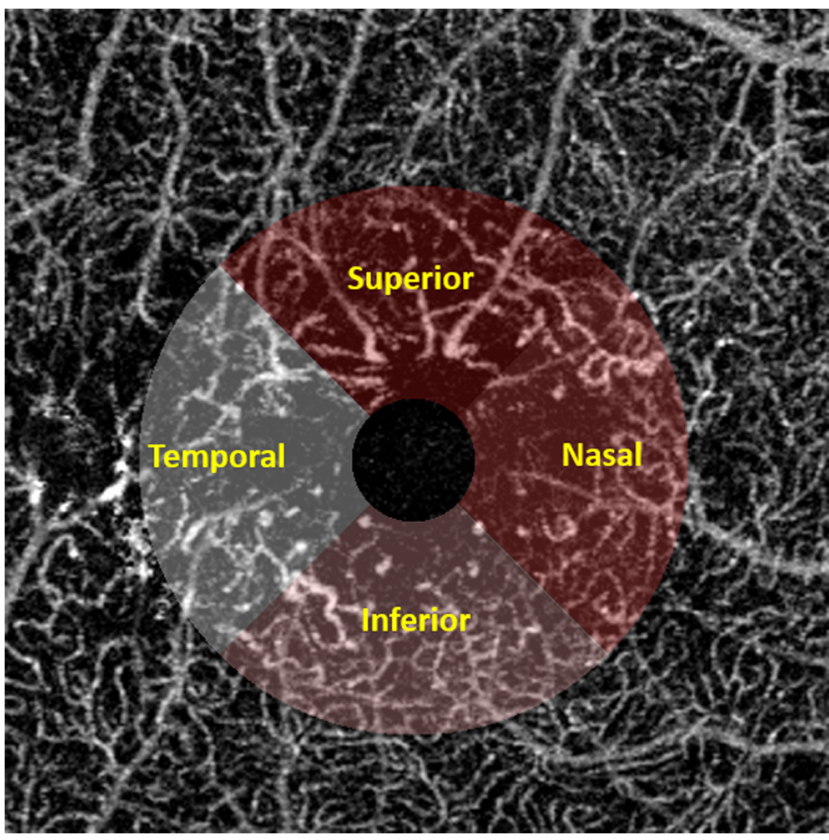

Fig. 12 Illustration of the quadratic analysis. 2-mm circle is selected excluding the FAZ. Superior, inferior, temporal, and nasal quadrants are labeled in different colors as showed in text.

\section{Discussion}

In this report, we proposed five metrics, VAD, VSD, VDI, VPI, and VCI, for the quantitative assessment of OCT angiograms. There are several important features in our analytical approach. First, the quantitative OCTA analysis using the five metrics is highly repeatable as the coefficients of variance are all smaller than 0.031, suggesting that the quantitative assessment at follow-up and when used for therapeutic monitoring should provide a high level of accuracy when using OCTA. We showcased repeatability using both a normal case and a BRVO case, with different FOV and scanning location. Second, the quantitative metrics are useful to provide clinicians and physicians with a rapid and comprehensive strategy for interpreting OCT angiograms. Unlike previous studies that only provided a single quantitative index such as VAD or skeleton density, our proposed quantitative analysis includes five indices, e.g., VAD, VSD, VDI, VPI, and VCI. Along with three quantitative maps, the vessel density map, the vessel diameter map, and the vessel complexity map, the information about vascular abnormalities could be visualized and extracted from OCTA images based on multiple perspectives. Vessel length, size, area, perimeter, and morphological complexity can be made all available in one step of image analysis, potentially helping clinicians understand OCTA images better. For example, in the case that we studied here, the five metrics showed its promise to distinguish BRVO from NPDR. Both of these two cases demonstrate VAD decrease. However, for BRVO, the decrease of VAD is dominated by skeleton decline; whereas for NPDR, both VDI and VSD are increased. Third, the quantitative metrics can provide additional information when presented alongside with the qualitative OCTA images.

One commonly acknowledged shortcoming of OCTA is that it is difficult to show hyperpermeability or leakage from abnormal vasculature as seen with FA. This difference between OCTA and FA originates from the fact that OCTA detects
Table 6 Quantitative analysis of a MacTel2 case receiving antiVEGF treatment.

\begin{tabular}{|c|c|c|c|c|}
\hline Indices & Quadrant & $1 / 9 / 2015$ & $2 / 5 / 2015$ & $3 / 31 / 2015$ \\
\hline \multirow[t]{4}{*}{ VDI } & Superior $^{\mathrm{a}}$ & 21.397 & 19.704 & 18.995 \\
\hline & Temporal $^{\mathrm{a}}$ & 20.228 & 18.857 & 18.063 \\
\hline & Inferior $^{a}$ & 19.901 & 19.082 & 18.268 \\
\hline & Nasal & 19.441 & 18.916 & 19.228 \\
\hline \multirow[t]{4}{*}{ VAD } & Superior & 0.391 & 0.368 & 0.341 \\
\hline & Temporal & 0.364 & 0.322 & 0.340 \\
\hline & Inferior & 0.401 & 0.378 & 0.395 \\
\hline & Nasal & 0.388 & 0.374 & 0.361 \\
\hline \multirow[t]{4}{*}{$\mathrm{VCl}$} & Superior & 4.615 & 4.952 & 4.852 \\
\hline & Temporal $^{\mathrm{a}}$ & 4.840 & 4.726 & 5.307 \\
\hline & Inferior $^{\mathrm{a}}$ & 5.313 & 5.378 & 5.830 \\
\hline & Nasal & 5.112 & 5.371 & 5.329 \\
\hline \multirow[t]{4}{*}{ VSD } & Superior & 0.097 & 0.095 & 0.092 \\
\hline & Temporal & 0.092 & 0.086 & 0.096 \\
\hline & Inferior & 0.105 & 0.103 & 0.107 \\
\hline & Nasal & 0.097 & 0.098 & 0.094 \\
\hline \multirow[t]{4}{*}{ VPI } & Superior & 0.225 & 0.221 & 0.213 \\
\hline & Temporal & 0.236 & 0.221 & 0.241 \\
\hline & Inferior & 0.264 & 0.257 & 0.271 \\
\hline & Nasal & 0.250 & 0.254 & 0.248 \\
\hline
\end{tabular}

${ }^{a} p<0.05$ in ANOVA test.

Table 7 Statistics of quantitative indices for normal subjects.

\begin{tabular}{ccccc} 
& Mean & Standard deviation & Lower 95\% & Upper 95\% \\
\hline VDI & 24.073 & 0.640 & 23.454 & 25.036 \\
VAD & 0.505 & 0.010 & 0.493 & 0.518 \\
VSD & 0.143 & 0.004 & 0.138 & 0.149 \\
VPI & 0.339 & 0.004 & 0.329 & 0.349 \\
VCI & 17962 & 591 & 17228 & 18696 \\
\hline
\end{tabular}

the movement of RBCs while FA detects the presence and leakage of a small molecular weight dye that remains mostly unbound to protein and capable of diffusing out from abnormal vessels. Though fluorescein leakage registers as an important marker of a vascular abnormality, it also prevents detailed visualization of microvasculature. In this study, we showed that with 
our quantitative maps, OMAG might also detect the regions of abnormal microvasculature that might correspond to areas of dye leakage on FA images. This abnormal vasculature possesses many abnormal characteristics that are probably associated with increased permeability, such as vascular dilation, a decrease of vascular complexity, and capillary drop out. While it is the current dogma that OCTA is not able to detect vascular hyper-permeability, this lack of leakage detection may be mitigated to some extent by our quantitative analysis because our results suggest that the regions of vascular dilation or decreasing complexity correlate well with leakages on FA. However, this finding is based on a limited number of clinical cases. Clearly, additional studies with larger sample sizes are needed to confirm this finding.

Moreover, our quantitative analysis is versatile in terms of selecting the ROIs. In this study, the analysis was reported on the entire image. Our algorithm also allows users to freely select only part of the OCT angiograms as a region of interest with a variety of useful choices, such as rectangle, circle, ring, or ellipse regions as shown in Figure 8. This feature may be useful when user wants to compare the same ROI of different scans as the scanned regions might vary. Furthermore, we have also demonstrated the software's ability in conducting quadratic analysis in both a BRVO case and a MacTel 2 case. Even though previous studies have shown the quantification of vessel density in normal and DR human subjects, ${ }^{16,17}$ to the best of our knowledge, this is the first study that vessel diameter and vessel complexity have been quantified on OCTA images with capillary level resolution. Our three quantitative maps together are useful for providing an integrated analysis on various ocular diseases. In addition, it should also be possible to combine all our quantitative maps together to yield an integrated "vascular abnormality indicator map". In order to do so, we would need to complete the normal database, upon which to combine the vessel density map, vessel diameter map, and vessel complexity map together, with appropriate weighting factors if necessary, to serve as a quick indicator to locate a region of vascular abnormality from patients' scans.

There are several limitations in our quantitative OMAG technology. First, the OCTA data shows projection artifacts in the deep retinal layers on both cross-sectional images and en face images. When photons interact with moving particles, the phase of the light wave changes as a result of the Doppler effect. Therefore, when the light travels through blood vessels in living tissue, only part of the light would reflect back during its first interaction with RBCs while the majority of the photons carry on that are influenced by their first interaction. As a result, OCTA signals in the deeper layers also carry the shadows of blood vessels from the superficial plexus. These projection artifacts from the retinal vasculature projected onto deeper layers of the retina are ubiquitous and difficult to notice unless the OCTA data is segmented into superficial and deep plexus and the superficial signals are removed from the deeper flow signals. The effects of the projection tails in retinal vasculature on quantitative analysis remain underexplored, but the inherent physical characteristics of these artifacts suggest that the effects should be consistent. As a consequence, we did not perform any correction on the projection tails in this study. Zhang et al. ${ }^{24}$ recently proposed a practical and automatic projection artifact removal algorithm, which has been demonstrated to successfully remove these projection artifacts efficiently for visualizing macular neovascularization. Work is currently in progress to incorporate this projection artifact removal algorithm into our quantification assessment in order to provide more accurate quantification of the retinal microvasculature in the deep retinal layers.

The second limitation of this study is the inherent trade-off between scanning time and sampling density that makes high resolution wide-field OCTA scans more time-consuming. Although there is also a single $6 \mathrm{~mm} \times 6 \mathrm{~mm}$ scanning protocol available with a lower sampling spacing between adjacent Ascans, we chose the montage scan mode over the single scan mode because the montage scan provides a much higher sampling spacing $(\sim 10 \mu \mathrm{m})$ and would not lose detailed information of the microvasculature. Last, we have not performed a reproducibility study on different OCT systems with the same subject. Since some of our quantitative indices are pixel based values, the comparison of different images will require the same pixel size.

In conclusion, we have demonstrated a comprehensive quantitative OCTA analysis package for normal and diseased eyes. Collectively, the VAD, VSD, VPI, VSD, and VCI can detect abnormal microvasculature and also provide repeatable quantitative results. Our vessel density map, vessel diameter map, and vessel complexity map jointly could be useful for detecting regions with vascular abnormalities that could also leak fluorescein on FA images. Overall, quantitative OCTA has promising future applications in research and clinical management of ocular diseases.

\section{Acknowledgments}

Research supported in part by grants from National Eye Institute (R01EY024158), Carl Zeiss Meditec, Inc. (Dublin, CA), an unrestricted grant from the Research to Prevent Blindness, Inc., New York, NY.

\section{References}

1. R. K. Wang et al., "Three dimensional optical angiography," Opt. Express 15(7), 4083-4097 (2007).

2. E. Moult et al., "Ultrahigh-speed swept-source OCT angiography in exudative AMD," Ophthalmic Surg. Lasers Imaging Retina 45(6), 496-505 (2014).

3. T. S. Hwang et al., "Optical coherence tomography angiography features of diabetic retinopathy," Retina 35(11), 2371-2376 (2015).

4. R. F. Spaide, "Volume-rendered optical coherence tomography of diabetic retinopathy pilot study," Am. J. Ophthalmol. 160(6), 1200-1210 (2015).

5. B. E. Kobrin Klein, "Overview of epidemiologic studies of diabetic retinopathy," Ophthalmic Epidemiol. 14(4), 179-183 (2007).

6. R. Klein et al., "The epidemiology of age-related macular degeneration," Am. J. Ophthalmol. 137(3), 486-495 (2004).

7. A. H. Kashani et al., "Optical coherence tomography angiography of retinal venous occlusion," Retina 35(11), 2323-2331 (2015).

8. Q. Zhang et al., "Swept source optical coherence tomography angiography of neovascular macular telangiectasia type 2," Retina 35(11), 2285-2299 (2015).

9. A. Mariampillai et al., "Speckle variance detection of microvasculature using swept-source optical coherence tomography," Opt. Lett. 33(13), 1530-1532 (2008).

10. Y. Jia et al., "Split-spectrum amplitude-decorrelation angiography with optical coherence tomography," Opt. Express 20(4), 4710-4725 (2012).

11. B. Braaf et al., "Angiography of the retina and the choroid with phaseresolved OCT using interval-optimized backstitched B-scans," Opt. Express 20(18), 20516-20534 (2012).

12. A. Zhang et al., "Methods and algorithms for optical coherence tomography-based angiography: a review and comparison," J. Biomed. Opt. 20(10), 100901 (2015). 
13. R. K. Wang et al., "Depth-resolved imaging of capillary networks in retina and choroid using ultrahigh sensitive optical microangiography," Opt. Lett. 35(9), 1467-1469 (2010).

14. C. L. Chen et al., "Optic nerve head perfusion in normal eyes and eyes with glaucoma using optical coherence tomography-based microangiography," Quant. Imaging. Med. Surg. 6(2), 125-133 (2016).

15. R. Reif et al., "Quantifying optical microangiography images obtained from a spectral domain optical coherence tomography system," $J$. Biomed. Imaging 2012, 9 (2012).

16. Y. Jia et al., "Quantitative optical coherence tomography angiography of vascular abnormalities in the living human eye," Proc. Natl. Acad. Sci. U. S. A. 112(18), E2395-E2402 (2015).

17. S. A. Agemy et al., "Retinal vascular perfusion density mapping using optical coherence tomography angiography in normals and diabetic retinopathy patients," Retina 35(11), 2353-2363 (2015).

18. Q. Zhang et al., "Wide-field imaging of retinal vasculature using optical coherence tomography-based microangiography provided by motion tracking," J. Biomed. Opt. 20(6), 066008 (2015).
19. X. Yin, J. R. Chao, and R. K. Wang, "User-guided segmentation for volumetric retinal optical coherence tomography images," J. Biomed. Opt. 19(8), 086020 (2014).

20. A. F. Frangi et al., "Multiscale vessel enhancement filtering," in Medical Image Computing and Computer-Assisted Interventation-MICCAI'98, pp. 130-137, Springer Verlag, Berlin, Germany (1998).

21. A. B. Watson, "Perimetric complexity of binary digital images," Math J. 14, 1-40 (2012)

22. P. J. Mackenzie and G. A. Cioffi, "Vascular anatomy of the optic nerve head," Can. J. Ophthalmol. 43(3), 308-312 (2008).

23. M. R. Thorell et al., "Swept-source OCT angiography of macular telangiectasia type 2," Ophthalmic Surg. Lasers Imaging Retina 45(5), 369380 (2014).

24. A. Zhang, Q. Zhang, and R. K. Wang, "Minimizing projection artifacts for accurate presentation of choroidal neovascularization in OCT microangiography," Biomed. Opt. Express 6(10), 4130-4143 (2015).

Biographies for the authors are not available. 